\title{
Multiple HPV infections in female sex workers in Western Kenya: implications for prophylactic vaccines within this sub population
}

Sonia Menon ${ }^{1,5^{*}}$, Davy van den Broeck ${ }^{1,4}$, Rodolfo Rossi ${ }^{3}$, Emilomo Ogbe ${ }^{1}$ and Hillary Mabeya ${ }^{1,2}$

\begin{abstract}
Background: Whilst the imputed role of High Risk (HR) HPV infection in the development of cervical lesions and cancer has been established, the high number of HPV genotypes that Female Sex workers (FSW) harbour warrants that the synergistic effects of potential HR (pHR) and HR HPV genotypes be elucidated to assess the potential impact of prophylactic vaccines. This population in Kenya also harbours a number of other vaginal infections and STIs, including bacterial vaginosis (BV), trichomonas vaginalis (TV) and candida spp.

The aims of this cross-sectional analysis in Kenya are to explore the epidemiology of abnormal cytology and the pairing of pHR/HPV genotypes in HIV-negative and HIV-infected FSW.

Methods: A cross-sectional study design of 616 FSW from Western Kenya aged between 18 and 61 years during 2009-2015 using a peer recruitment sampling strategy.

Results: Of the 599 FSW who underwent cytological examination, 87 had abnormal cytology (14.5\%; 95\% Cl: 12.017.6\%). A combined prevalence of HPV16 and 18 (29.6\%; 95\% Cl: 22.2-37.8\%) was observed in abnormal cytology. HPV 53 and 51 were the most observed pairing in FSW with abnormal cytology. Significant adjusted associations were found between abnormal cytology and TV (aOR: 30; 95\% Cl: 14.1-62.9), multiple HR HPV (aOR: 3.7; 95\% Cl: 1.97.3), HPV 51 (aOR 3.7; 95\% Cl 1.6-8.6) and HPV 52 (aOR 6.1; 95\% Cl: 2.8-13.3).

Conclusion: HPV 51 and 52 were independently associated with abnormal cervical cytology in both HIV negative/ positive FSW. The strong association between TV and cervical dysplasia and the high percentage of FSW harbouring more than one STI underscore the need for enhanced STI management within the framework of cervical cancer prevention.
\end{abstract}

Keywords: FSW, HIV, High risk HPV, Potential high risk HPV, Multiple pHR/HR coinfections, Vaccine efficacy

\section{Background}

Human Papilloma Viruses (HPV) are double-stranded DNA viruses which are now deemed to be the chief etiological agents in cervical intraepithelial neoplasias and cancers [1]. High risk (HR) genotypes, which are associated with cervical cancer include HPV genotypes 16, 18, 31, 33, $35,39,45,51,52,56,58,59,66,68$ while HPV types 26 ,

\footnotetext{
* Correspondence: soniasimonemenon@gmail.com

${ }^{1}$ International Centre for Reproductive health, Department of Obstetrics and Gynaecology, Ghent University, De Pintelaan 185 P3, 9000 Ghent, Belgium

${ }^{5} \mathrm{CDC}$ Foundation Atlanta, Atlanta, USA

Full list of author information is available at the end of the article
}

$53,67,70,73$ and 82 are now classified as possible carcinogenic, and low risk (LR) HPV genotypes considered benign include $6,11,42,43$, and 44 [2]. The $15 \mathrm{HR}$ oncological viral strains, which have been identified can be broken down into different species: the HPV 16 group (alpha-9) of the alpha-papillomavirus genus (HPV 31, HPV33, HPV 35, HPV 52, and HPV 58) and the HPV 18 group (alpha-7) (HPV39, HPV 45, HPV 59, and HPV 68).

Cervical intraepithelial neoplasia (CIN) can be histologically graded into mild dysplasia (CIN 1), moderate dysplasia (CIN 2), and severe dysplasia to carcinoma in situ (CIN 3). Several studies have reported a robust 
association between HIV and HPV co-infection and therefore the development of CIN and genital cancer, [3, 4] along with a persistence and recurrence of preinvasive cervical lesions, CIN 2 or CIN 3 [5].

It is well recognized that among the $14 \mathrm{HR}$ HPV genotypes, HPV 16 and 18 are associated with approximately two thirds of all invasive cervical carcinomas [6]. After HPV16/18, data confirm HPV31/33/35/45/52/58 as the most frequently detected genotypes in Invasive Cervical Cancer (ICC) worldwide [7, 8].

Prophylactic vaccines against HPV 16 and 18 are currently being rolled out across the globe for the prevention of cervical cancer, which is likely to yield a significant impact on the future burden of cervical cancer, particularly in sub Saharan Africa, where screening is scarce. Although the bivalent/ quadrivalent vaccines, including the LR HPV 6 and 11 constitute a crucial milestone in cervical cancer prevention in HIVnegative women, epidemiological data available suggest that in HIV positive populations, HPV 16 has shown to be frequent, but not as predominant as seen in most HIV negative populations [9, 10]. Moreover, HIV immunosuppression has been linked to multiple HPV infection [11, 12]. Concomitant infection with multiple HPV genotypes has been found to be attributable to the inability to clear HPV infections as well as to the reactivation of latent HPV infections; both occurring as a consequence of immune suppression [13, 14].

Also, epidemiological knowledge of pHR HPV types is highly limited, mainly because commercial molecular assays focus on HR HPV genotypes. There is a paucity of data on these genotypes in HIV positive women with abnormal cytology, notwithstanding their potential enhanced role in cervical dysplasia development in HIV positive patients $[15,16]$.

In Kenya, the Ministry of Public Health and Sanitation has developed a comprehensive cervical cancer prevention strategy, entailing plans for administrating quadrivalent vaccine to preteen girls in the near future. Currently they are running the pilot programme Kituwi in Eastern Kenya and awaiting approval for nationwide rollout and successful global funding [17]. The not yet commercialized nonavalent vaccine in Kenya, containing additional HPV types HPV 6, 11, 16, 18, 31, 33, 45, 52, and 58 antigens may have the ability to prevent $90 \%$ of ICC cases worldwide.

In Kenya, as in many parts of sub-Saharan Africa, where the penal code specifically penalizes prostitution [18], FSW bear the greatest burden of HIV and STI infections. Concomitant STIs and vaginal infections may lead to prolonged HPV infection, which may in turn increase the risk of CIN $[19,20]$. Bacterial vaginosis (BV) and Trichomonas vaginalis (TV), have been associated with an increased risk of squamous intraepithelial lesions and/or CIN based on biopsy results $[21,22]$.

As early as 1985, a study reported that HIV prevalence was as high as $61 \%$ among a group of FSW in Nairobi
[23]. A recent study reported that in Kenya, 5\% of the urban female population of reproductive age could be sex workers [24].

The objectives of this analysis were primarily to assess genotype-specific distribution of $\mathrm{pHR} / \mathrm{HR} \mathrm{HPVs}$ in FSW with abnormal cytology, as well as the pairing prevalence of certain $\mathrm{pHR} / \mathrm{HR}$ HPV genotypes found in HIV negative and HIV infected women with abnormal cytology; secondly, to investigate which HPV genotypes and other variables were associated with abnormal cytology.

\section{Methods \\ Study design}

A cross-sectional design was used to explore associations between abnormal cytology and pHR/HR HPV genotypes. This cross-sectional study based on record reviews adhered to the methodological guidelines recommended in the STROBE document on observational studies [25].

Women were excluded if they were pregnant, $<18$ years of age, had a history of cervical dysplasia or cancer, had current abnormal bleeding or bloody discharge, and/or had a hysterectomy. Snowball sampling was undertaken instead of randomized sampling, which is an often used strategy for locating difficult-to-reach and stigmatized populations [26]. This involved a sample of women engaged in sex work being recruited through informational gatherings, snowball sampling and neighborhood outreach. Inclusion criteria for the study entailed being female, giving consent after being explained the objectives of the study, and having engaged in sex in exchange for money, goods, services, or drugs in the last three months. In order to reduce friendship bias, a limit of referral of 10 FSW was established. This activity was undertaken by means of an extensive community outreach program by Gynocare Women and Fistula Hospital, two nongovernmental organizations specializing in reproductive health, to identify women with obstetric fistula, STI screening and cervical screening in Western Kenya. The screening was supported by Gent University, Belgium.

\section{Sample size}

The sample size was calculated to allow for a prevalence of at least $15 \%$ for abnormal cytology, [27] with a confidence interval of $95 \%$ and a power of $80 \%$.

\section{Data collection}

\section{Structured questionnaire}

A structured paper questionnaire was privately administered by trained interviewers covering socio-demographic characteristics, and sexual behavior. Participants were offered testing for HIV and HPV. HIV results were disclosed to participants and women infected with HIV received counseling and treatment. 


\section{Specimen collection and laboratory testing}

A gynaecological examination was performed using a swab. Candida colonization was diagnosed by Gram stain; bacterial vaginosis was scored according to Nugent's criteria. Infection with Trichomonas vaginalis was diagnosed by PCR, using a validated method which was part of the HPV genotyping essay.

A HIV diagnosis was performed using rapid immunoassays: Uni-Gold ${ }^{\mathrm{Tm}}$ Recombigen $^{\bullet}$ HIV (Trinity Biotech plc, Bray, Ireland) and Determine ${ }^{\circ}$ HIV-1/2 (Abbott Japan co Ltd, Minato-Ku, Tokyo, Japan). In the case of indeterminate results, an enzyme-linked immunosorbent assay was used to confirm HIV status.

\section{Biologic specimens}

Cervical samples were collected using a cervix brush (Cervex-brush ${ }^{\oplus}$, Rovers ${ }^{\oplus}$, Oss, The Netherlands), and cervical cytology was assessed with conventional Papanicolaou (Pap) smears. Slides were read by a cytologist with master level training, supervised by a pathologist. An external cytopathologist provided quality control. The Bethesda Reporting System was used for cytological classification [28].

The cervix brush tips were preserved in a liquid-based cytology collection medium (SurePath ${ }^{\circ}$, Tripath Imaging Inc., Burlington, North Carolina, USA) and stored at $4{ }^{\circ} \mathrm{C}$ until further processing.

\section{Ethical approval}

Ethical approval for the study was obtained from the Institutional Research and Ethics Committee at the MOI University in Kenya (No 000187) on August 11 ${ }^{\text {th }}, 2011$.

\section{Statistics and data analysis}

Data analysis was done using STATA version 12 (StataCorp LP, College Station, TX). Due to incomplete information about the study samples, we checked whether the missing data (10\%) were randomly distributed by performing the Little's MCAR test. Continuous variables were then converted into categorical. Age was dichotomized into $\geq 30$ years and $<30$ years; this categorization was used to reflect the WHO 2014 guideline concerning cervical screening. The number of $\mathrm{pHR} / \mathrm{HR}$ HPV co-infections was also dichotomized as a categorical variable with 1 and $\geq 2$ genotypes.

We first described the distribution of $\mathrm{pHR} / \mathrm{HR}$ HPV types observed among women with both normal cytology and abnormal cytology, for which the overall prevalence and $95 \%$ confidence intervals (95\% CI) based upon normal distributions were calculated.

To examine patterns of clustering of high-risk HPV types, the prevalence of $\mathrm{pHR} / \mathrm{HR}$ HPV genotypes in presence of another pHR/HR HPV genotypes by abnormal cytology was calculated, which was defined as the proportion of women with abnormal cytology who were positive for the $\mathrm{pHR} / \mathrm{HR}$ HPV genotypes. The prevalence of pairings detected in women with HSIL was also calculated.

The variables were explored by means of tabulation and cross-tabulation. The $\chi^{2}$ test was used to assess whether there was an association between CIN 2+ and various risk factors. In building the regression models, age, STIs and multiple pHR/HR HPV genotypes tested were entered. A multivariable logistic regression analysis was performed to assess the association between pHR/HR HPV genotypes and abnormal cytology, ASC-US or higher, the main outcome of interest and to simultaneously control for potential confounders. The Likelihood Ratio Test (LRT) was used to measure the association of each variable with the outcome.

To assess for a possible interaction due to age, logistic regression models were fitted with and without the interaction term; significance for interaction was then checked through visual inspection of the OR and LRT. Statistical significance was considered at $p \leq 0.05$.

\section{Results}

Out of the 616 participants, data from only 599 participants could be analysed because of the quality of the specimen. Missing values were observed for BV (61), TV (7) and candida spp (7). The Little's MCAR test revealed that the missing values were randomly distributed among the participants $(p=0.4)$, therefore unlikely to have introduced information bias.

The mean age of study participants was 28 years and median parity was 2 (range: 0-7 children). Regular condom use, which was defined as always or almost always was reported in $21.7 \%$ of 369 women. (95\% CI $=17.6-26.2 \%$ ). The median number of partners in the past week was 4 (IQR: 2-7). Table 1 depicts the prevalence of categories for age and sexual behavior.

Of the $616 \mathrm{FSW}$ who underwent cytological examination, 512 (85.5\%: 95\% CI: 82.4-88.2) had normal cytology, 87 had abnormal cytology (14.5\%; 95\% CI: 12.0-17.6\%) and 17 were excluded due to poor quality of the sample, leaving 599 participants on whom we could perform the analysis. Of the FSW population, $192 \mathrm{FSW}$ were HIV positive, of

Table 1 Reports the prevalence of categories for age and sexual behaviour

\begin{tabular}{ll}
\hline $\begin{array}{l}\text { Socio demographic variables } \\
>30 \text { years }\end{array}$ & Percentage (95\% Cl) \\
$\leq 30$ years & $40.1 \%(95 \% \mathrm{Cl}: 36.2-44.1)$ \\
Sexual behavior: & $59.9 \%(95 \% \mathrm{Cl}: 55.9-63.8)$ \\
$>4$ sexual partners the past week & $1.9 \%(95 \% \mathrm{Cl}: 1.0-3.3)$ \\
$\leq 4$ sexual partners & $98.1 \%(95 \% \mathrm{Cl}: 96.7-99.0)$ \\
Regular use of condom & $21.7 \%(95 \% \mathrm{Cl} 17.6-26.2)$ \\
No regular use of condom & $78.3 \%(95 \% \mathrm{Cl}: 73.8-92.3)$ \\
\hline
\end{tabular}


which $27.1 \%$ (95\% CI: 20.9-34.0\%) had abnormal cytology. Table 2 in annex reports the prevalence of cervical abnormalities observed in the sample $(N=616)$.

The prevalence of $\mathrm{pHR} / \mathrm{HR} \mathrm{HPV}$ and multiple $\mathrm{pHR} / \mathrm{HR}$ co-infections in the 616 FSW was 57.7 and $32.8 \%$ respectively. In HPV infected FSW, HIV-infected FSW had a significantly higher number of co-infections (2.0) than HIV negative FSW (0.9), $p<0.001$.

The prevalence of BV in this population was $48.3 \%$, followed by TV $31.4 \%$, and candida spp $19.9 \%$. FSW with BV had the highest prevalence of multiple pHR/HR coinfections with $53.4 \%$, followed by TV, and candida spp, 38.8 and $23.9 \%$ respectively. Table 3 reports the prevalence of each HPV genotypes and vaginal infections/TV.

Normal and abnormal cytology and each HPV genotypes The combined prevalence of HPV 16 and HPV 18 was $27.1 \%$, and of the two pHR HPV genotypes tested, HPV 53 and 66, 20.8\% (95\% CI: 17.6-24.2\%). In women with abnormal cytology, we observed a multiple pHR/HR HPV genotype prevalence of $65.5 \%$. See Table 4 in annex, which reports the prevalence of each HPV genotypes.

\section{Potential HR HPV in abnormal cytology}

HPV 53 and HPV 66 were found as a stand-alone pHR HPV genotype in two LSIL cases.

\section{Pairings of pHR/HR HPV genotypes in abnormal cytology}

A higher prevalence of pairings in abnormal cytology were observed in HIV infected FSW than HIV negative FSW. The most frequently observed pairings in HIV- negative FSW were HPV 18 and $31(n=3)$ occurrences, and HPV 31 and $52(n=2)$, involving genotypes phylogenetically related to the HPV 16.

In HIV-infected women, HPV 53 and HPV 51 are observed in 5 of the most prevalent pairings, followed by HPV 16, observed in 4 of the most prevalent pairings. Whilst in HIV negative FSW, the most prevalent coinfection pairings exhibited a mixed alpha 9-alpha 7 combination or a homogenous alpha 9 pattern, in HIV infected women, the non-alpha 9/7 pHR HPV genotypes, HPV 53 (alpha 6) and HPV 51 (alpha 5) figure prominently. See Table 5 in annex which depicts the most prevalent pairing occurrences in women with abnormal cytology and HSIL.

Table 2 Reports the prevalence of cervical abnormalities observed in the sample $(N=616)$

\begin{tabular}{lll}
\hline Cytological status & $\mathrm{n}$ & \% of FSW (95\% Cl) \\
\hline Normal cytology & 512 & $85.5 \%(82.4-88.2)$ \\
ASC-US & 10 & $1.7 \%(0.8-3.04)$ \\
LSIL & 63 & $10.5 \%(8.2-13.3)$ \\
HSIL & 14 & $2.3 \%(12.8-3.9)$ \\
Excluded samples due to poor quality & 17 & $2.8 \%$ \\
\hline
\end{tabular}

Table 3 Reports the prevalence of each HPV genotypes and vaginal infections/TV

\begin{tabular}{llll}
\hline pHR/HR HPV Genotype & Frequency $(n)$ & $\begin{array}{l}\text { Percentage } \\
(N=616)\end{array}$ & $95 \% \mathrm{Cl}$ \\
HPV $16(N=616)$ & 99 & $16.10 \%$ & $13.3 \%-19.2 \%$ \\
HPV $18(N=616)$ & 68 & $11.04 \%$ & $08.7 \%-13.8 \%$ \\
HPV $31(N=616)$ & 49 & $8.00 \%$ & $5.9 \%-10.4 \%$ \\
HPV $33(N=616)$ & 2 & $0.30 \%$ & $0.04 \%-1.2 \%$ \\
HPV $35(N=616)$ & 70 & $11.40 \%$ & $9.0 \%-14.1 \%$ \\
HPV $39(N=616)$ & 48 & $7.80 \%$ & $5.8 \%-10.2 \%$ \\
HPV $51(N=615)$ & 52 & $8.50 \%$ & $3.7 \%-7.4 \%$ \\
HPV $53(N=616)$ & 68 & $11.04 \%$ & $8.7 \%-13.8 \%$ \\
HPV $56(N=616)$ & 45 & $7.30 \%$ & $5.4 \%-9.7 \%$ \\
HPV 58 $(N=616)$ & 30 & $4.90 \%$ & $3.3 \%-6.9 \%$ \\
HPV $59(N=616)$ & 75 & $12.20 \%$ & $9.7 \%-15.02 \%$ \\
HPV 66 $(N=616)$ & 60 & $9.70 \%$ & $7.5 \%-12.4 \%$ \\
HPV 68 $(N=616)$ & 9 & $4.90 \%$ & $3.3 \%-6.9 \%$ \\
Vaginal infections and TV & & & \\
BV $(N=555)$ & 268 & $48.30 \%$ & $44.06 \%-52.5 \%$ \\
TV $(N=609)$ & 191 & $31.4 \%$ & $27.6 \%-35.2 \%$ \\
Candida $(N=609)$ & 121 & $19.90 \%$ & $16.8 \%-23.3 \%$ \\
\hline
\end{tabular}

Risk factors for abnormal cytology

In the univariate analysis, age did not appear to be associated with abnormal cytology: women under 30 years of age have a crude OR 1.1 (95\% CI: $0.7-1.8 p=0.6)$ of having abnormal cytology compared to older women.

Table 4 Reports the prevalence of each HPV genotype in women with abnormal cytology

\begin{tabular}{llll}
\hline HPV genotype & Abnormal cytology & $\%(N=87)$ & $95 \% \mathrm{Cl}$ \\
\hline HPV 16 & 24 & $28.6 \%$ & $19.2-39.5$ \\
HPV 18 & 15 & $17.9 \%$ & $10.0-27.0$ \\
HPV 31 & 13 & $15.5 \%$ & $8.5-25.0$ \\
HPV 33 & 1 & $1.2 \%$ & $0.03-6.5$ \\
HPV 35 & 18 & $21.4 \%$ & $13.2-31.7$ \\
HPV 39 & 13 & $15.5 \%$ & $8.5-25.0$ \\
HPV 45 & 9 & $10.7 \%$ & $5.0-19.4$ \\
HPV 51 & 18 & $21.4 \%$ & $13.2-31.7$ \\
HPV 52 & 26 & $31.0 \%$ & $21.3-42.0$ \\
HPV 53 & 21 & $25.0 \%$ & $16.2-35.6$ \\
HPV 56 & 13 & $15.5 \%$ & $8.5-25.0$ \\
HPV 58 & 4 & $13.3 \%$ & $3.8-30.7$ \\
HPV 59 & 17 & $23.6 \%$ & $14.4-35.1$ \\
HPV 66 & 9 & $10.7 \%$ & $5.0-19.4$ \\
HPV 67 & & & \\
HPV 68 & 4 & $4.8 \%$ & $1.3-11.7$ \\
\hline
\end{tabular}


Table 5 Most prevalent pairing occurrences in women with abnormal cytology and HSIL

\begin{tabular}{lll}
\hline Prevalent pairings in abnormal cytology & $\begin{array}{l}\text { Occurrences } \\
\text { in HIV-negative women }\end{array}$ & $\begin{array}{l}\text { \% in abnormal } \\
\text { cytology }\end{array}$ \\
HPV 18 and 31 & 2 & 3 \\
HPV 31 and 52 & 7 & 2 \\
Prevalent pairings in HIV infected women with abnormal cytology \\
HPV 16 and 39 & 2 & 6 \\
HPV 16 and 52 & 9 & 7 \\
HPV 16 and 51 & 4 & 5 \\
HPV 16 and 53 & 10 & 7 \\
HPV 18 and 52 & 12 & 5 \\
HPV 18 and 53 & 8 & 5 \\
HPV 31 and 51 & 2 & 5 \\
HPV 35 and 51 & 2 & 5 \\
HPV 35 and 53 & 4 & 7 \\
HPV 45 and 53 & 0 & 6 \\
HPV 45 and 59 & 2 & 5 \\
HPV 51 and 53 & 2 & 7 \\
HPV 51 and 56 & 1 & 6 \\
HPV 52 and 56 & 3 & 6 \\
HPV 53 and 56 & 1 & 5 \\
\hline
\end{tabular}

A very strong significant association was found between TV and abnormal cytology OR: 30.0 (95\% CI: 14.1-62.9) adjusting for age and pHR/HR HPV genotypes, BV and candida spp and HIV.

A statistically significant OR, adjusted for age was found for multiple HPV and abnormal cytology compared to single HPV genotype infection. This association decreased but remained significant (OR; 3.9; $p<0.001$; 95\%CI: 1.9-7.8) when adjusted for HIV.

Whilst all pHR/HR HPV genotypes were significant predictors of abnormal cytology, when adjusted for age pHR/ HR HPV genotypes co-infections, HIV, BV, TV, and candida spp these associations became statistically insignificant, except for HPV 51 and 52. Table 6 in annex depicts the age, BV, TV and candida spp adjusted association between specific pHR/HR HPV genotypes and abnormal cytology.

As no interaction terms were significant, no result reflecting the differential impact for that particular outcome was presented.

\section{Discussion}

\section{Summary of results}

In the present study, we observed a high prevalence of women harbouring more than two pHR/HR HPV genotypes, which was significantly higher in HIV-1-infected women, consistent with results of several studies illustrating that HIV-1-infected women not only have a greater prevalence of HR-HPV infection but multiple coinfections [29, 30].

Our observations are in agreement with those of a large study on multiple HPV infections in Costa Rica in which young healthy women with multiple infections were at significantly increased risk of CIN 2+, Although our findings of an association between TV and abnormal cytology are congruent with other observations [31] demonstrating an association between TV and abnormal cytology, [32, 33] our study suggest an unprecedented strong association.

After adjusting for age and multiple pHR/HR HPV coinfections, BV, TV and candida spp, no significant association was observed between any single pHR/HR HPV and abnormal cytology, except for HPV 51 and 52 in both HIV negative and positive women. This is incongruent with our previous findings in an exclusively HIV-infected study population in Belgium, [34] in which only the association between HPV 39 and abnormal cytology became statistically significant.

In contrast to HPV 31 and 58 being the most observed frequent pairing in Brazil in HIV infected women, and HPV 31 and 66 and HPV 39 and 52 in our Belgian study population, in this FSW study population, HPV 31, 52 and 66 do not figure prominently within pairings observed in HIV-infected women despite a higher prevalence of HPV 52 than HPV 53.

Also, our high prevalence of women with abnormal cytology without any detected HPV genotypes can be attributed to our testing of only 18 genotypes out of the over 200 HPV types described. Whilst some types may be inducing low grade lesions, they may also lack a cancer-initiating capacity.

\section{Strengths and limitations}

Our major strength was that all cervical smears were histologically confirmed and the high sensitivity of the HPV DNA diagnostics employed. However, whilst our sample was large, the number of FSW with abnormal cytology was small, which precluded us from exploring particular co-infection patterns as a risk factor. Moreover, due to our lack of behavioral and clinico-epidemiological data, including smoking, CD4 count, HAART use or the presence of other co-infections, we have not been able to adjust for these potential confounders, nor assess whether these factors were associated with cervical abnormalities. Also, there may be potential for selection bias as FSW with similar characteristics may have been sampled, as a result of the convenience sampling method used. However, a convenience sampling strategy may have inadvertently excluded those FSW operating in a more clandestine fashion and thereby not benefitting from a social network.

A limitation related to a cross sectional study design may be the lack of data concerning age of acquisition of 
Table 6 Association between vaginal infections, TV, specific pHR/HR HPV genotypes and abnormal cytology; OR from Logistic regression; $p$-value from LRT

\begin{tabular}{|c|c|c|c|c|}
\hline Vaginal infections and STIs & OR Model $1(95 \% \mathrm{Cl})$ & $p$-value & OR Model 2 (95\% Cl) & $p$-value \\
\hline BV & $0.9(0.6-1.5)$ & 0.8 & $0.8(0.5-1.4)$ & 0.5 \\
\hline TV & $24.8(12.7-48.3)$ & $<0.001$ & $30.0(14.1-62.9)$ & $<0.001$ \\
\hline Candida spp & $1.0(0.5-1.7)$ & 1.0 & $0.9(0.5-1.7)$ & 0.7 \\
\hline Multiple HPV infection & $5.3(2.9-9.7)$ & $<0.001$ & $3.7(1.9-7.3)$ & $<0.001$ \\
\hline HPV 16 & $1.9(0.8-4.5)$ & 0.1 & $1.2(0.5-3.2)$ & 0.5 \\
\hline HPV 18 & $0.8(0.3-2.1)$ & 0.7 & $1.04(0.4-2.8)$ & 0.9 \\
\hline HPV 31 & $0.5(0.2-1.5)$ & 0.2 & $0.6(0.2-1.7)$ & 0.3 \\
\hline HPV 33 & $3.9(0.05-293.9)$ & 0.5 & $2.8(0.03-254.6)$ & 0.6 \\
\hline HPV 35 & $1.3(0.6-3.0)$ & 0.5 & $1.1(0.5-2.7)$ & 0.9 \\
\hline HPV 39 & $3.3(1.3-8.7)$ & 0.03 & $2.5(0.9-7.1)$ & 0.09 \\
\hline HPV 51 & $3.7(1.6-8.6)$ & 0.002 & $3.7(1.5-9.0)$ & 0.004 \\
\hline HPV 52 & $6.1(2.8-13.3)$ & $<0.001$ & $4.0(1.6-8.2)$ & 0.002 \\
\hline HPV 53 & $2.0(0.8-4.9)$ & 0.1 & $1.4 ;(0.5-3.8)$ & 0.5 \\
\hline HPV 56 & $2.5(1.0-6.6)$ & 0.06 & $2.0(0.7-5.7)$ & 0.2 \\
\hline HPV 58 & $0.9(0.2-3.6)$ & 0.9 & $1.1(0.3-5.2)$ & 0.9 \\
\hline HPV 66 & $1.2(0.5-3.0)$ & 0.7 & $1.0(0.4-3.0)$ & 0.9 \\
\hline HPV 68 & $1.7(0.2-17.0)$ & 0.7 & $0.8(0.1-7.4)$ & 0.8 \\
\hline
\end{tabular}

Model 1: OR adjusting for age, pHR/HR HPV genotypes, vaginal infections/TV

Model 2: OR adjusting for age and pHR/HR genotypes, vaginal infections/TV and HIV

HIV infection since it is possible this may have occurred too late in life for some of the women in our study to influence abnormal cytology. Similarly, an analysis of a cross sectional study for exploring associations between multiple HPV genotypes and abnormal cytology may have inherent limitations as infections may have been acquired concurrently or sequentially, therefore, resulting in the criterion of temporality for causation not being met. This may have an impact as immunologic responses may differ following concurrent acquisition of multiple HPV genotypes from infections that are acquired sequentially.

\section{Implications for vaccination programs}

The only significant association observed between abnormal cytology and HPV 51 and HPV 52 underscore the need for post quadrivalent and nonavalent vaccine surveillance in both HIV negative and HIV infected FSW.

In light of a high prevalence of multiple HR HPV infections in this FSW HIV-negative and infected population, it will need to be elucidated whether cross protection may be hampered by the additional burden due to other synergistic relationships among HR HPV genotypes present. A recent systematic review and metaanalysis [35] found that bivalent Cervarix $@$ vaccine from GlaxoSmithKline had better cross protection against HPV 31 in persistent infection, but that efficacy against persistent infections with types 31 appeared to decrease with longer follow-up, suggesting a waning of crossprotection. It still remains to be determined whether a cross protection can be extrapolated to HIV-infected women and in presence of multiple HR HPV genotypes.

Moreover, with a high prevalence of the pHR HPV 53 in pairings with the vaccine preventable HPV 16 and HPV 18 in HIV infected women, it will need to be determined how HPV 53 will fare within the vacuum that ensues the quadrivalent vaccine [36]. In addition, given the high median number of $\mathrm{pHR} / \mathrm{HR}$ HPV genotypes harboured by this population, the synergies between not only two pHR/HR HPV genotypes need to be determined but within a context of other prevalent genotypes, capable of inducing cervical cancer genesis. Whether Gardasil and Cervarix can attain a $70 \%$ reduction of cervical cancer may be contingent upon the natural history of the imputed pHR/HR HPV genotypes in cancer genesis along with their synergistic effects.

Our very high association between TV and cervical dysplasia underscores the need for STI management to be integrated within cervical cancer prevention program. Furthermore, the biological interaction between TV and $\mathrm{HPV}$ and its subsequent capacity to induce progression of cervical dysplasia should be further explored. Moreover, the impact of immune modulating infections, such as tuberculosis, malaria and helminthic infections on cervical disease progression should be elucidated in HIV triple co-infected women with HPV and TV. 


\section{Conclusion}

Co-infection with pHR/HR HPV genotypes was more strongly associated with abnormal cytology than any single high-risk HPV. In light of the high prevalence of multiple pHR/HR HPV genotypes harboured by FSW and especially HIV infected women, its micro epidemiology in cervical carcinoma in HIV positive women needs to be explored in order for the vaccine efficacy to be assessed.

Whilst the quadrivalent vaccine may be effective in reducing the prevalence of abnormal cytology in HIV negative and HIV infected FSW, the high presence of multiple infections with HPV 16 requires that the micro epidemiology of concurrent be elucidated. In particular, the high prevalence of the non alpha 9 and 7 genotypes, HPV 51 and HPV 53 observed in pairings with HPV 16 and HPV 18 in HIV infected FSW requires further characterization.

These current gaps in epidemiology underscore the need for FSW, HIV negative or positive to be regularly monitored in the post quadrivalent/nonavalent vaccine era.

The strong association observed between TV and cervical dysplasia as well as the high percentage of FSW harbouring more than one vaginal infection/STI begs for the elucidation of synergistic interactions between multiple STIs to be better assessed as factor(s) for cervical dysplasia and for a wider encompassing cervical cancer prevention framework.

\section{Abbreviations}

ASC-H: Typical squamous cells-cannot exclude high-grade squamous intraepithelial lesion; ASC-U: Atypical cells of undetermined significance; FSW: Female sex workers; HIV: Human immunodeficiency virus; HPV: Human Papilloma virus; HSIL: High grade squamous intraepithelial lesion; LR HPV: Low risk HPV; LSIL: Low grade squamous intraepithelial lesion; pHR HPV: Potential high risk

\section{Acknowledgement}

Dr Stacy Harmon and Dr Mbabazi Kariisa for their editing, critical feedback of the intellectual content. This research received no specific grant. HM is supported by VLIR-UOS.

\section{Funding}

Our research was funded by the VLIR IUC Moi University.

\section{Availability of data and materials}

Yes.

\section{Authors' contributions}

SM lead author conceived and drafted the manuscript, performed the statistical analysis, and interpreted the findings. DB participated in the conception of the paper, revision and validation of the article. RR participated in the data analysis, interpretation of findings, and in the revision of the article. EO: participated in the data analysis, interpretation of findings, and in the revision of the article. HM: participated in designing the study, interpretation of findings and in the revision of the article. All authors read and approved the final manuscript.

\section{Competing interests}

The authors declare that they have no competing financial, political, personal, religious, ideological, academic, intellectual, commercial or any other interests.

\section{Consent for publication}

Yes.

Ethics approval and consent to participate

Informed consent was sought by all participants. Ethical approval for the study was obtained from the Institutional Research and Ethics Committee at the MOI University in Kenya.

\section{Author details}

${ }^{1}$ International Centre for Reproductive health, Department of Obstetrics and Gynaecology, Ghent University, De Pintelaan 185 P3, 9000 Ghent, Belgium. ${ }^{2}$ Moi University/Gynocare Fistula Centre, Eldoret, Kenya. ${ }^{3}$ AMBIOR (Applied Molecular Biology Research Group), Antwerpen, Belgium. ${ }^{4}$ Faculty of Medicine and Health Sciences, Laboratory of Cell Biology \& Histology, University of Antwerp, Antwerp, Belgium. ${ }^{5}$ CDC Foundation Atlanta, Atlanta, USA.

Received: 15 June 2016 Accepted: 21 December 2016

Published online: 06 January 2017

\section{References}

1. Ng'andwe C, Lowe JJ, Richards PJ, Hause L, Wood C, Angeletti PC. The distribution of sexually-transmitted Human Papillomaviruses in HIV positive and negative patients in Zambia, Africa. BMC Infect Dis. 2007;7:77. doi:10. 1186/1471-2334-7-77.

2. Bouvard V, Baan R, Straif K, Grosse Y, Secretan B, El Ghissassi F, Benbrahim-Tallaa L, Guha N, Freeman, Galichet L, Cogliano V, WHO International Agency for Research on Cancer Monograph Working Group. A review of human carcinogens_-Part B: biological agents. Lancet Oncol. 2009;10:321-2.

3. Hawes SE, et al. Increased risk of high-grade cervical squamous intraepithelial lesions and invasive cervical cancer among African women with human immunodeficiency virus type 1 and 2 infections. J Infect Dis. 2003;188(4):555-63.

4. De Sanjose S, Diaz M, Castellsague $X$, et al. Worldwide prevalence and genotype distribution of cervical human papilloman DNA in women with normal cytology: a meta-analysis. Lancet Infect Dis. 2007;7:453-9.

5. Russomano F, Paz BR, Camargo MJ, Grinstejn BG, Friedman RK, Tristao MA Oliveira CA. Recurrence of cervical intraepithelial neoplasia in human immunodeficiency virus-infected women treated by means of electrosurgical excision of the transformation zone (LLETZ) in Rio de Janeiro, Brazil. Sao Paulo Med J. 2013;131(6):405-10. doi:10.1590/1516-3180.2013.1316578.

6. Bosch FX, de Sanjose S. Chapter 1: Human papillomavirus and cervical cancer: burden and assessment of causality. J Natl Cancer Inst Monogr. 2003:31:3-13.

7. Li N, Franceschi S, Howell-Jones R, Snijders PJ, Clifford GM. Human papillomavirus type distribution in 30,848 invasive cervical cancers worldwide: Variation by geographical region, histological type and year of publication. Int J Cancer. 2011;128(4):927-35.

8. Rahman M, Sasagawa T, Yamada R, Kingoro A, Ichimura H, Makinoda S. High prevalence of intermediate-risk human papillomavirus infection in uterine cervices of kenyan women infected with human immunodeficiency virus. J Med Virol. 2011;83:1988-96.

9. Didelot-Rousseau MN, Nagot N, Costes-Martineau V, Valles X, Ouedraogo A, Konate I, Weiss HA, Van de Perre P, Mayaud P, Segondy M, Yerelon Study Group. Human papillomavirus genotype distribution and cervical squamous intraepithelial lesions among high-risk women with and without HIV-1 infection in Burkina Faso. Br J Cancer. 2006;95:355-62.

10. Ahdieh $\mathrm{L}$, Klein RS, Burk R, et al. Prevalence, incidence, and type-specific persistence of human papillomavirus in Human Immunodeficiency Virus (HIV)-positive and HIV-negative women. J Infect Dis. 2001;184:682-90.

11. Levi JE, Kleter B, Quint WG, Fink MC, Canto CL, Matsubara R, et al. High prevalence of human papillomavirus (HPV) infections and high frequency of multiple HPV genotypes in human immunodeficiency virus-infected women in Brazil. J Clin Microbiol. 2002:40:3341-5.

12. Moscicki AB, Ellenberg JH, Farhat S, Xu J. Persistence of human papillomavirus infection in HIV-infected and -uninfected adolescent girls: risk factors and differences, by phylogenetic type. J Infect Dis. 2004;190:37-45.

13. Strickler HD, Burk RD, Fazzari M, Anastos K, Minkoff H, Massad LS, Hall C, Bacon M, Levine AM, Watts DH, Silverberg MJ, Xue X, Schlecht NF, Melnick S, Palefsky JM. Natural history and possible reactivation of human papillomavirus in human immunodeficiency virus-positive women. J Natl Cancer Inst. 2005;97:577-86.

14. Palefsky JM, Minkoff H, Kalish LA, Levine A, Sacks HS, Garcia P, Young M, Melnick S, Miotti P, Burk R. Cervicovaginal human papillomavirus infection in 
human immunodeficiency virus-1 (HIV)-positive and high-risk HIV-negative women. J Natl Cancer Inst. 1999;91:226-36.

15. Schopp B, Holz B, Zago M, Stubenrauch F, Petry KU, Kjaer SK, et al. Evaluation of the performance of the novel PapilloCheck HPV genotyping test by comparison with two other genotyping systems and the HC2 test. J Med Virol. 2010;82:605-15.

16. Barcellos RB, Almeida SE, Sperhacke RD, Verza M, Rosso F, Medeiros RM, et al. Evaluation of a novel microplate colorimetric hybridization genotyping assay for human papillomavirus. J Virol Methods. 2011;77:38-43.

17. Friedman AL, Oruko KO, et al. Preparing for human papillomavirus vaccine introduction in Kenya: implications from focus-group and interview discussions with caregivers and opinion leaders in Western Kenya. BMC Public Health. 2014;14:855.

18. International Models project on women's right. Current Legal Framework: Prostitution in Kenya. 2011. http://www.impowr.org/content/current-legalframework-prostitution-kenya. Accessed 8 May 2016.

19. Finan RR, Tamim H, Almawi WY. Identification of Chlamydia trachomatis DNA in human papillomavirus (HPV) positive women with normal and abnormal cytology. Arch Gynecol Obstet. 2002;266(3):168-71.

20. Syrjanen K, Mantyjarvi R, Vayrynen M, et al. Chlamydial cervicitis in women followed-up for human papillomavirus (HPV) lesions of the uterine cervix. Acta Obstet Gynecol Scand. 1985;64:467-71.

21. Platz-Christensen JJ, Sundstrom E, Larsson PG. Bacterial vaginosis and cervical intraepithelial neoplasia. Acta Obstet Gynecol Scand. 1994;73:586-8.

22. Noel JC, Fayt I, Romero Munoz MR, et al. High prevalence of high-risk human papillomavirus infection among women with Trichomonas vaginalis infection on monolayer cytology. Arch Gynecol Obstet. 2010;282:503-5.

23. Ngugi EN, Plummer FA, Simonsen JA, Cameron DW, Bosire M, et al. Prevention of transmission of human immunodeficiency virus in Africa: effectiveness of condom promotion and health education among prostitutes. Lancet. 1988; 2(8616):887-90. doi:10.1016/50140-6736(88)92480-4.

24. Odek WO, Githuka GN, Avery L, Njoroge PK, Kasonde L, Gorgens M, et al. Estimating the size of the female sex worker population in Kenya to inform HIV prevention programming. PLoS One. 2014;9(3):e89180. doi:10.1371/ journal.pone.0089180.

25. von Elm E, Altman DG, Egger M, Pocock SJ, Gotzsche PC, Vandenbroucke $J P$, the STROBE initiative. The Strengthening the Reporting of Observational Studies in Epidemiology (STROBE) statement: guidelines for reporting observational studies. Lancet. 2007;370:1453-7.

26. Ulin PR, Robinson ET, Tolley EE. Qualitative methods in public health: A field guide for applied research. San Francisco: APA (6th ed.) Jossey-Bass; 2005.

27. Musa J, Achenbach C, Taiwo B, et al. High-risk human papilloma virus and cervical abnormalities in HIV-infected women with normal cervical cytology. Infect Agents Cancer. 2014;9:36.

28. Davey DD. Cervical cytology classification and the Bethesda System. Cancer J. 2003;9(5):327-34

29. Grinsztejn B, Veloso VG, Levi JE, Velasque L, Luz PM, et al. Factors associated with increased prevalence of human papillomavirus infection in a cohort of HIV-1-infected Brazilian women. Int J Infect Dis. 2008:13(1):72-80.

30. Temmerman M, Tyndall MW, Kidula N, Claeys P, Muchiri L, et al. Risk factors for human papillomavirus and cervical precancerous lesions, and the role of concurrent HIV-1 infection. Int J Gynaecol Obstet. 1999;65(2):171-81.

31. Yap EH, Ho TH, Chan YC, et al. Serum antibodies to Trichomonas vaginalis in invasive cervical cancer patients. Genitourin Med. 1995;71:402-4.

32. Donders GGG, Depuydt CE, Bogers J.P, Vereecken AJ. Association of Trichomonas vaginalis and Cytological Abnormalities of the Cervix in Low Risk Women. Kaul R, ed. PLoS ONE. 2013;8(12):e86266. doi:10.1371/journal.pone.0086266.

33. Viikki M, Pukkala E, Nieminen P, Hakama M. Gynaecological infections as risk determinants of subsequent cervical neoplasia. Acta Oncol. 2000;39:71-5.

34. Menon S, Rossi R, Benoy I, Bogers JP, van den Broeck D. Human papilloma virus infection in HIV-infected women in Belgium: implications for prophylactic vaccines within this subpopulation. Eur J Cancer Prev. 2016 [Epub ahead of print].

35. Malagón T, Drolet M, Boily MC, Franco EL, Jit M, Brisson J, Brisson M. Crossprotective efficacy of two human papillomavirus vaccines: a systematic review and meta-analysis. Lancet Infect Dis. 2012:12(10):781-9. doi:10.1016/ S1473-3099(12)70187-1.

36. Padalko E, Ali-Risasi C, Van Renterghem L, Bamelis M, De Mey A, Sturtewagen $Y$, Vastenavond $H$, Vanden Broeck D, Weyers S, Praet M. Evaluation of the clinical significance of human papillomavirus (HPV) 53. Eur J Obstet Gynecol Reprod Biol. 2015;191:7-9. doi:10.1016/j.jogrb.2015.04.004.

\section{Submit your next manuscript to BioMed Central and we will help you at every step:}

- We accept pre-submission inquiries

- Our selector tool helps you to find the most relevant journal

- We provide round the clock customer support

- Convenient online submission

- Thorough peer review

- Inclusion in PubMed and all major indexing services

- Maximum visibility for your research

Submit your manuscript at www.biomedcentral.com/submit
Biomed Central 